\title{
Preliminary in vivo evaluation of a needle insertion manipulator for central venous catheterization
}

\author{
Yo Kobayashi" ${ }^{1 *}$ Ryutaro Hamano ${ }^{1}$, Hiroki Watanabe ${ }^{1}$, Takuma Koike ${ }^{1}$, Jaesung Hong ${ }^{2}$, Kazutaka Toyoda ${ }^{3}$, \\ Munenori Uemura ${ }^{3}$, Satoshi leiri ${ }^{3}$, Morimasa Tomikawa ${ }^{3}$, Takeshi Ohdaira ${ }^{3}$, Makoto Hashizume ${ }^{3}$ and Masakatsu G Fujie ${ }^{1}$
}

\begin{abstract}
Central venous catheterization is associated with potential complications secondary to accidental puncture, including venous bleeding and pneumothorax. We developed a system that avoids these complications and simplifies the procedure using a robot to provide puncture assistance. We herein report a puncture experiment conducted in vivo in a porcine to evaluate the manipulator. The right and left jugular veins of a pig were punctured five times each through both opened and unopened skin at a puncture angle and speed. A venous placement rate of $80 \%$ was obtained with opened skin. A much lower rate of $40 \%$ was obtained with unopened skin. One of five attempts in opened skin was unsuccessful, likely because of the stick-slip phenomenon. This system was effective for jugular venous puncture of opened skin. Future studies should focus on puncture conditions that facilitate needle placement, inhibit the stick-slip phenomenon, and minimize needle bending due to the presence of skin.
\end{abstract}

Keywords: Central venous catheterization; Needle insertion; Surgical robot

\section{Background}

Central venous catheterization (CVC) is a technique that involves venous puncture and insertion of a catheter to perform transfusions. CVC is used in a broad spectrum of cases in which the presence of a reliable line close to the heart is essential to administer fluids ranging from highly concentrated nutritional supplements to vasoactive medications. For CVC, the vein is penetrated by a puncture needle with an outer sheath (for example, $18 \mathrm{G}, \phi 2.4 \mathrm{~mm}$ ). The backflow of venous blood is confirmed after puncture to ensure that the needle has been successfully inserted into the vein. The needle is subsequently removed, leaving only the outer sheath in place. The catheter is then inserted into the outer sheath at a predetermined length to reach the target site. Finally, the outer sheath is removed, and the catheter is sutured to the skin and fixed in place. During this series of steps, potential complications include bleeding caused by accidental puncture of an artery running parallel to the vein and pneumothorax caused by accidental puncture of the pleural membrane. In addition, it takes considerable

\footnotetext{
* Correspondence: you-k@fuji.waseda.jp

${ }^{1}$ Faculty of Science and Engineering \& Research Institute for Science and Engineering, Waseda University, 59-309, Ohkubo, Shijuku, Tolyo 169-8555, Japan Full list of author information is available at the end of the article
}

skill to ensure that the needle is inserted completely and that it is stopped at an appropriate point within the vessel. Therefore, our research focuses on puncture procedures for CVC, which is associated with a risk of complications and requires a high skill level. Specifically, the goal of this study is to develop a system that avoids these complications and simplifies the procedure using a robot that provides puncture assistance. We aimed to develop a robotic system to ensure a safer procedure.

\section{Related research}

To date, various types of robotic systems that assist needle insertion have been researched, and a number of manipulators and systems that assist puncture have been developed. Fichtinger et al. and Masamune et al. developed a prostate biopsy robot equipped with a function that uses X-ray computed tomography (CT) images to guide the catheter during surgery [1,2]. Loser et al. developed a needle insertion system in which the needle position is controlled using CT fluoroscopy images [3]. Yanof et al. developed a CT-guided robot for interventional procedures with preoperative and intraoperative planning [4]. Masamune et al. developed a small robotic system in which a magnetic resonance imaging (MRI) machine controls needle orientation in a 
narrow gantry space [5]. Hashizume et al. developed an MRI-guided surgical robotic system for minimally invasive laparoscopic surgery using a needle insertion manipulator [6]. Krieger et al. developed a remotely actuated manipulator for access to prostate tissue under MRI guidance [7]. Terayama et al. developed a curved multi-tube device that combines a straight needle and a needle with a curved section rather than the conventional straight needle and reported that by pushing out the inner needle with the curved section, it was possible to change the advancement direction of the needle inside the organ [8]. Fichtinger et al. developed a puncture-assist robot with ultrasound guidance for use during procedures involving the prostate gland [9], whereas Boctor et al. developed a computerassisted radiofrequency ablation (RFA) system that tracks an ultrasound probe supported by a passive mechanical arm when performing RFA using 3D ultrasound images [10]. Bassan et al. designed robotic systems for percutaneous procedures during prostate brachytherapy [11]. The authors of the current paper also developed a robotic system with an integrated ultrasound probe designed for use during needle insertion [12,13], as well as a punctureassist robot that supports MRI [14].

\section{Objectives and scope of this paper}

The needle insertion manipulator described in related research were designed to achieve accurate insertion of the needle into the center of tissues such as tumors within an organ. In contrast, with $\mathrm{CVC}$, the tip of the needle should stop inside the blood vessel, which requires a method to detect the direction of blood vessel orientation, and the needle must be inserted at an appropriate angle in relation to that orientation. The diameter of the target vein in this study was approximately $10 \mathrm{~mm}$, which is relatively small in relation to the diameter of the needle used for the puncture (for example, $18 \mathrm{G}, \phi 2.4 \mathrm{~mm}$ ). To ensure successful insertion of the needle into the vein, development of a robot with high positioning accuracy was necessary. Moreover, because CVC is frequently conducted at accident sites and other such locations, the device must be more compact and more convenient than the puncture devices described in previous related research. The device described here is more compact for use in ambulances and is more accurate for use during CVC. For this purpose, we developed a manipulator for ultrasoundguided needle insertion into a vein [15]. The paper focuses on describing only the development of a manipulator and a preliminary laboratory experiment to evaluate the accuracy of the manipulator.

A preliminary laboratory experiment in the previous work [15] focused only on the specification from engineering point of view, for example accuracy of the manipulator with the force-free environment. The further evaluation of the manipulator with the setup close to actual clinical situation is needed before clinical application. Specifically, evaluation using actual living organ is necessary because there are differences in the hardness and cutting properties of blood vessels and muscles between living organ and phantoms. In this point of view, animal studies of the developed manipulator is important issue for the evaluation.

Thus, we herein report a puncture experiment conducted in vivo in a porcine to evaluate the manipulator proposed in our earlier research [15]. Specifically, a robot system was used to puncture the jugular vein, which is typically used for CVC. We evaluated whether needle placement in the vein was possible using this system and identified problems in the robot system that would require improvement.

\section{Methods}

\section{Needle insertion manipulator}

Puncture was performed using the needle insertion manipulator shown in Figure 1. It measured approximately $100 \times 180 \times 60 \mathrm{~mm}$ and weighed approximately $700 \mathrm{~g}$. The experimental results indicate an accuracy of $1.0 \mathrm{~mm}$ or better, which is considered feasible for venous puncture. The major specifications for the manipulator are shown in Table 1. A force sensor was installed at the base of the needle so that the reaction force could be measured during puncture. The system detects the entry of the needle into the vein using these reaction force data and has the ability to stop the needle inside the vein. An easily configurable diagnostic ultrasound system viewable in real time was also used as a means of assisting the position based on the force sensor. The manipulator, which included an integral ultrasound probe, was able to position the needle tip at any point on the imaging plane. The positions of the needle tip was calculated based on the angles of each joint of the manipulator measured by encoders attached to the motors via the manipulator kinematics.

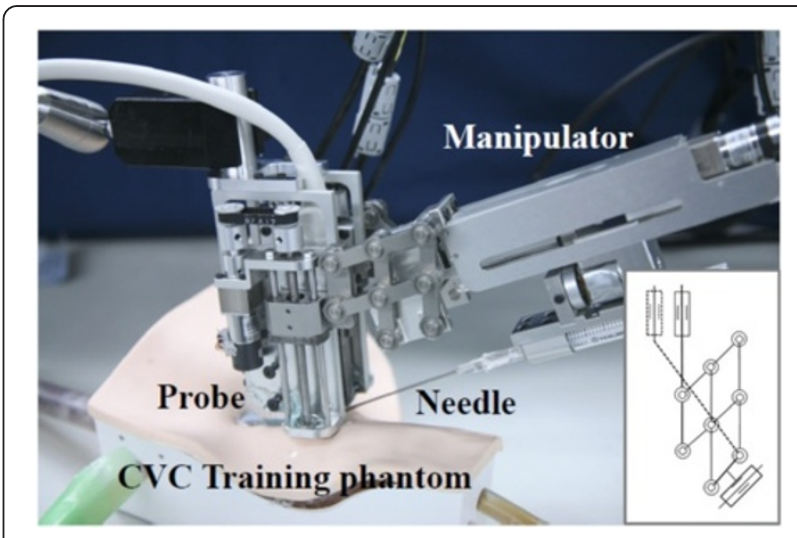

Figure 1 Needle insertion manipulator for central venous catheterization. 
Table 1 Major specifications of the manipulators

\begin{tabular}{cccc}
\hline Positioning accuracy in ultrasound image & Positioning accuracy in free-space & Size & Weight \\
\hline$\sim 1[\mathrm{~mm}]$ & $\sim 0.1[\mathrm{~mm}]$ & $\fallingdotseq 100 \times 180 \times 60[\mathrm{~mm}]$ & $\fallingdotseq 700[\mathrm{~g}]$ \\
\hline
\end{tabular}

These data summarize the data in this paper, which were substantiated in an earlier article [15].

\section{Experimental conditions}

After consultation with a surgeon, the porcine was chosen as the experimental species because its blood vessels have physical values (e.g., hardness) and geometric dimensions similar to those of humans. For this experiment, we chose a female domesticated pig weighing $30 \mathrm{~kg}$. The in vivo experiment was conducted with the approval of the Ethics Committee of Kyushu University Hospital. Appropriate care was taken to avoid any psychological or physiological distress on the animal during the experiment.

\section{Puncture site}

The jugular vein was chosen as the puncture site because it is one of the veins used as a catheter insertion route during CVC. The jugular veins run up the left and right sides of the neck, and the blood flow from the left side unites with that from the right side to reach the heart via the central veins. For this reason, puncture and catheter insertion into the right jugular vein involves a shorter route from the starting point of catheter insertion to the central veins and thus results in a higher insertion success rate in CVC. We were informed by the surgeon that puncture of the right jugular vein is performed more frequently than puncture of the left in CVC because of this anatomical feature. We therefore chose the right jugular vein as the first candidate puncture site and the left jugular vein as the second candidate puncture site.

Furthermore, because the jugular vein has an approximately 120 -mm puncturable length, we were able to perform several punctures in a single vein. However, during routine venous puncture, blood clots can form centrally along the puncture site as a result of the application of external force on the blood vessels, disturbance of blood flow secondary to the effect of the needle, and so forth. These blood clots can grow over time to occlude blood vessels, making puncture difficult. We therefore decided to start at a central puncture site on the jugular vein and

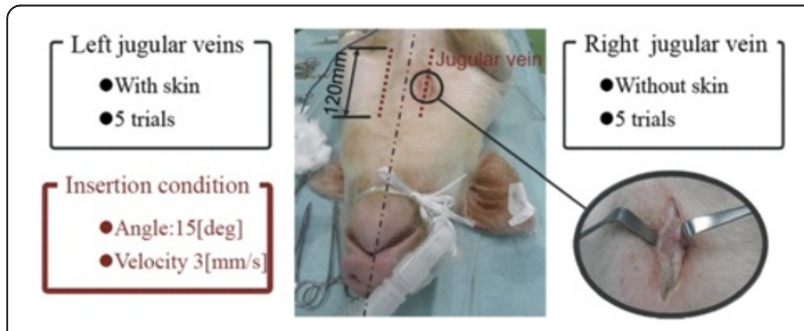

Figure 2 Experimental setup. gradually shift peripherally, performing five punctures in total. The puncture sites are shown in Figure 2.

\section{Effects of skin}

According to the surgeon, the hardness of human skin can be broadly differentiated. The condition of skin has a major effect on puncture difficulty and an important factor that must be taken into account when performing percutaneous puncture. Porcine skin (approximately $2 \mathrm{~mm}$ thick) is similar in thickness to human skin (mean thickness, $1-1.5 \mathrm{~mm}$ ), but is harder than the skin of an elderly human. In our experiment, it was anticipated that the presence of skin would affect one evaluation parameter: whether the needle had been placed in the vein. We therefore performed punctures under two conditions: with the skin opened beforehand and with the skin left intact. In the former condition, the skin was opened using an electric scalpel with the assistance of the surgeon. Figure 2 presents an expanded view of the opened section of skin.

\section{Experimental procedures and methods}

A puncture angle of $15^{\circ}$ and a puncture speed of $3 \mathrm{~mm} / \mathrm{s}$ were chosen. These conditions produced the most marked reduction in reaction force during the puncture of blood vessels in our previous research [16]. Five punctures each were performed on the jugular veins on the left and right sides. The puncture site was shifted from central to peripheral to avoid the effects of blood clots. The experimental process is described below.

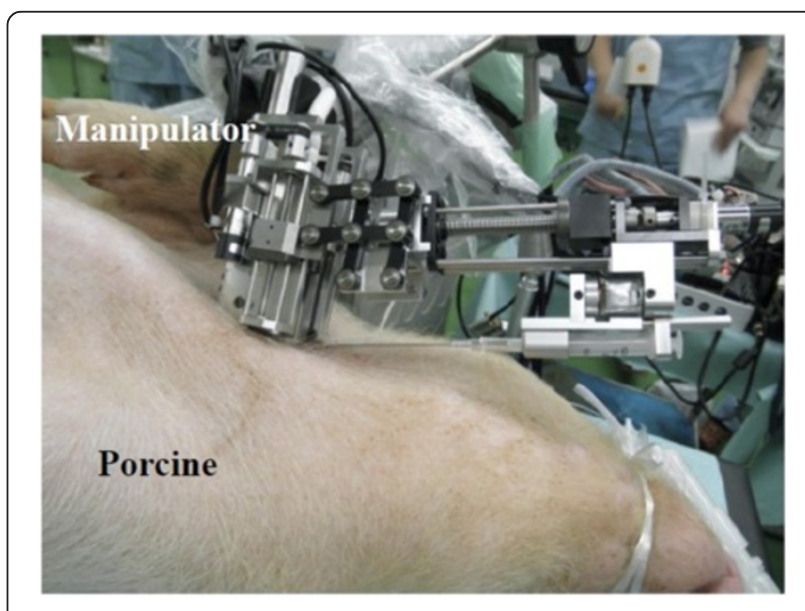

Figure 3 Overview of experiment with opened skin. 


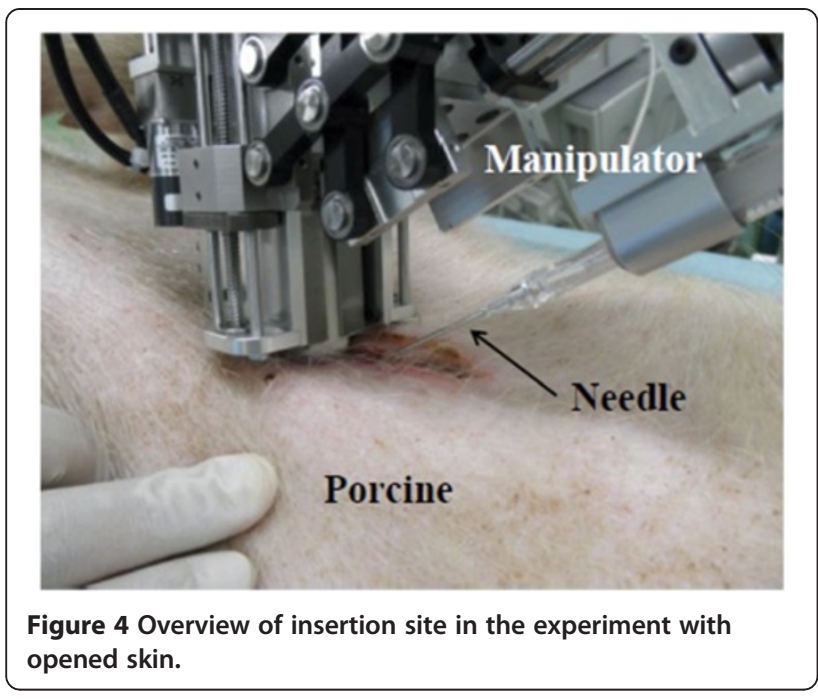

1. The operator holds the manipulator with the integral probe and captures the vein in the imaging plane.

2. With the vein captured, the manipulator is locked in place by the arm holding the endoscope.

3. The operator inputs a vein travel line on the ultrasound image.

4. The manipulator adopts a puncture angle of $15^{\circ}$ relative to the vein and begins to puncture at $3 \mathrm{~mm} / \mathrm{s}$.

5. The surgeon judges the moment at which the vein has been entered and stops the needle inside the vein on the basis of the ultrasound images and puncture reaction force data.

6. The surgeon decides whether intravenous placement was successful based on the ultrasound images.

\section{Results}

\section{Puncture through opened skin}

The arrangement of the venous puncture manipulator and the experimental animal during the experiment are

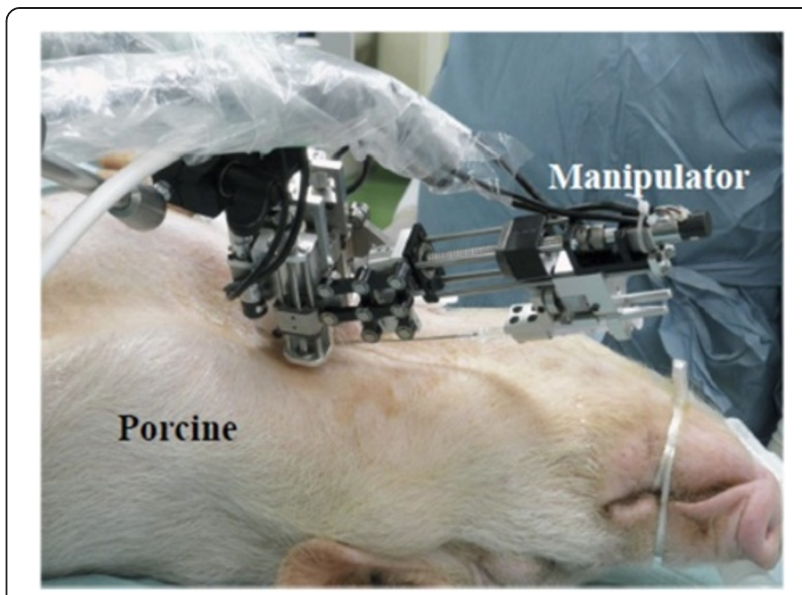

Figure 6 Overview of experiment with unopened skin.

shown in Figure 3 and 4, respectively. An example image captured by the diagnostic ultrasound equipment during puncture is shown in Figure 5. This image was captured with the diagnostic ultrasound equipment in the B-mode using a sector-scanning ultrasound probe. The scan depth was $50 \mathrm{~mm}$. The image represented by the broken red line at time:00 [sec] is a longitudinal image of the target right jugular vein. At time:02 [sec], the needle tip appears from the left of the screen. At time:04 [sec], the needle has moved while deflecting the upper wall of the vein downward on the screen. At time:06 [sec], the needle tip touches and begins to push the vein. At time:08 [sec], the needle tip has broken through the venous wall and entered the vein. At time:10 [sec], it is evident that the needle tip was stopped inside the vein. The needle placement success rate was $80 \%$. In one of the five puncture attempts, the needle was not successfully placed in the vein because the surgeon confirmed a drop in puncture reaction force and stopped the manipulator before the needle had actually entered the vein.
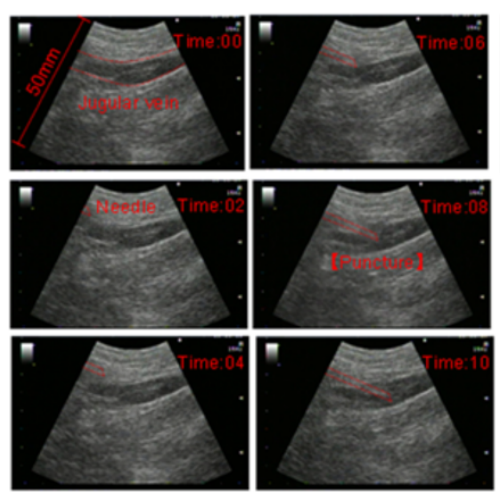

(a)Image with additional information
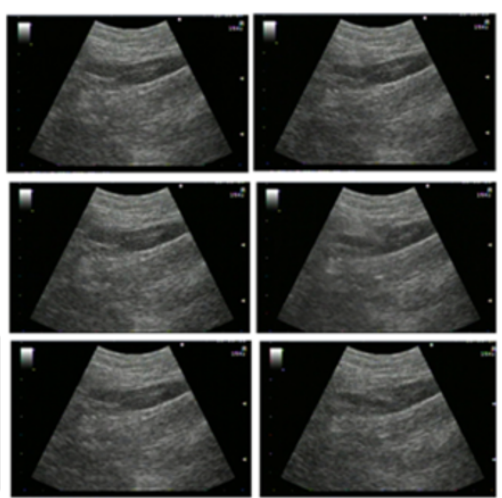

(b) Raw image

Figure 5 Ultrasound image at venous puncture in the experiment with opened skin. 


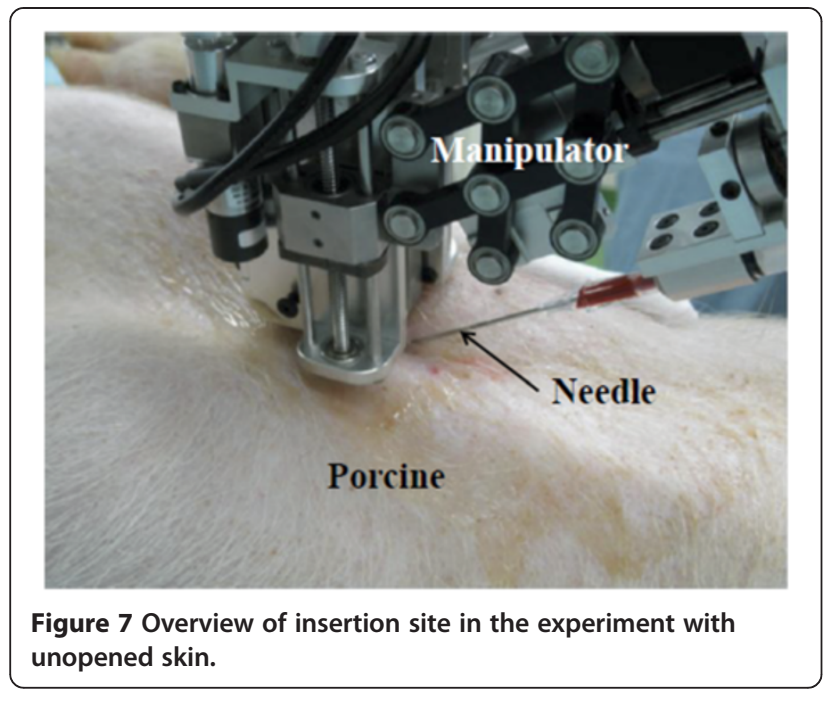

\section{Puncture through unopened skin}

The arrangement of the venous puncture manipulator and the experimental animal during the experiment are shown in Figure 6 and 7, respectively. An example image captured by the diagnostic ultrasound equipment during puncture is shown in Figure 8. This image was captured in the B-mode using a sector-scanning probe, as in experiment 1 (puncture through open skin). The scan depth was $50 \mathrm{~mm}$. The image represented by the broken red line at time:00 [sec] in Figure 8 is a longitudinal image of the target left jugular vein. The skin made it difficult for the probe to achieve close contact with the body surface, and the left side of the screen was not completely captured. At time:02 [sec], the needle tip appears from the left of the screen. At time:04 [sec], the needle approaches the upper wall of the vein. At time:06 [sec], the needle tip has begun to push the vein. At time:08 [sec], the needle tip has penetrated the venous wall and entered the vein. At time:10 [sec], the needle tip was stopped inside the vein. The needle placement success rate was $40 \%$. With the skin left unopened, the needle greatly deforms the skin before breakthrough, and at the same time the needle itself was seen to undergo bending.

\section{Reaction force during needle insertion}

Figures 9 and 10 shows the relation between needle displacement and reaction force during the experiments. Figure 9 shows the sample of the reaction force in the puncture through opened skin. Figure 10 shows the sample of the reaction force in the puncture through unopened skin. There are nonlinear increase in force.

\section{Discussion}

\section{Needle insertion manipulator}

When setting the posture of the manipulator before puncture, in some cases the puncture unit came in contact with the body surface if the angle relative to the body surface was shallow. To prevent interference between the puncture unit and the body, the section to which the needle is attached must be more compact. In some cases, the needle also stopped before the vein because of the insufficient range of motion in the manipulator section responsible for puncture. In humans, the jugular vein is located no more than $10 \mathrm{~mm}$ below the skin, whereas in the experimental setup, it was approximately $20 \mathrm{~mm}$ below the skin. The venous puncture manipulator used in this experiment was configured on the basis of human anatomy and therefore had an insufficient range of motion, particularly toward the center, where the veins sit more deeply below the skin. It would be possible to overcome this problem in the manipulator configuration by building and using two devices, as appropriate, with a common mechanism: one with a larger range of motion for experiments using pigs, and the other with a more restricted range of motion for use in humans.
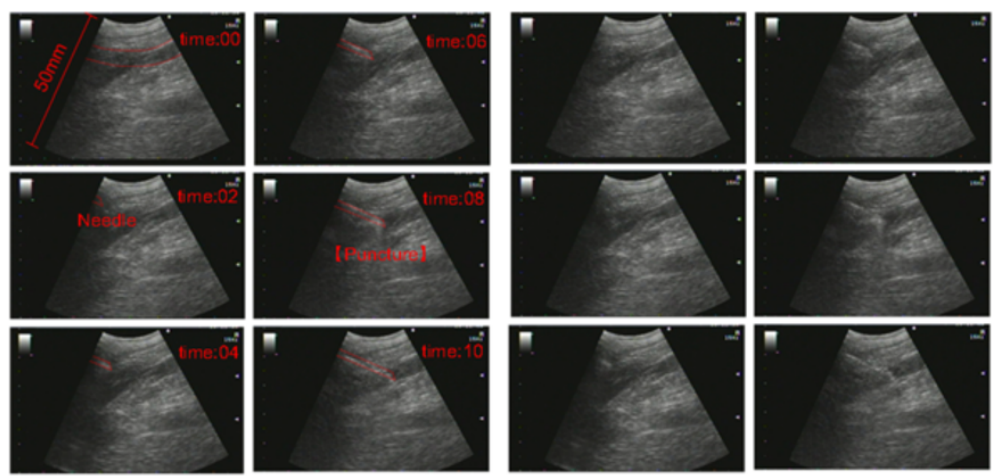

(a)Image with additional information

(b) Raw image

Figure 8 Ultrasound image at venous puncture in the experiment with unopened skin. 

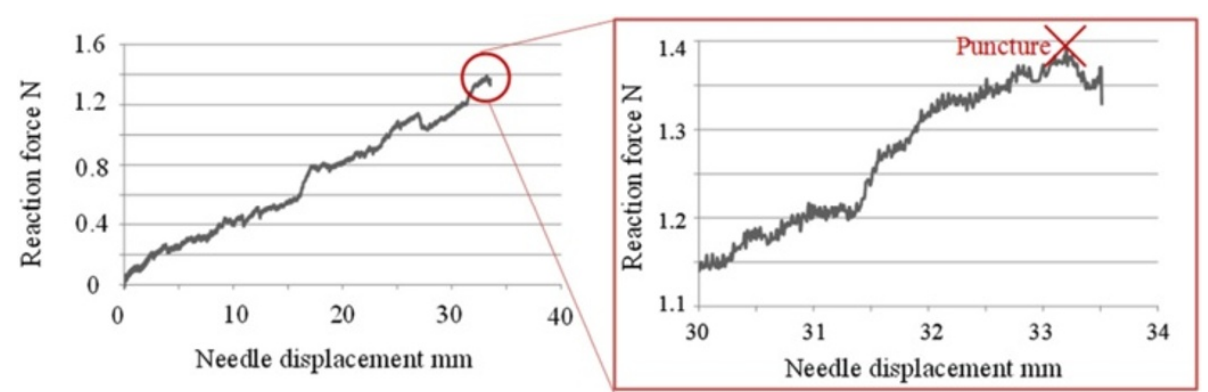

Figure 9 The relation between needle displacement and reaction force in the puncture through opened skin.

\section{Puncture through opened skin}

With the skin opened, very little bending occurred when the needle broke through the skin surface, and the vein could be reached without disturbing the intended puncture angle. This contributed to a high success rate for placement in the vein. The accident rate in clinical practice is reportedly approximately $20 \%$ and insertions are repeatedly carried out to position needle tip into vessel in the procedure. The complication risk become high when the needle penetrate a vessel. The manipulator stop the needle tip in the vessel or before the vessel. These result support that the system have certain advantage to prevent the risk of complication.

Failures occurred at the stage before the needle reached the vein, during which stick-slip was visually confirmed between the needle sides and surrounding tissue (fat and muscle). The stick-slip phenomenon produces a wavelike rise and fall in reaction force, and it appears that the surgeon saw this waveform and mistook it as a sign of needle entry into the vein. This problem could be solved by determining the rough depth of the vein at the time of input of the vein travel line on the ultrasound image and activating the entry detection function when this depth is approached. We are planning to install such a feature on the system in the near future.

\section{Puncture through unopened skin}

Failure to enter the vein during skin breakthrough may have been caused by large deformations of the skin that led to insertion point errors and by bending of the needle that resulted in deviation from the anticipated insertion pathway. Solving this problem requires both the development of a highly rigid needle that does not bend during skin breakthrough and the testing of needle tips to determine which shape produces the lowest maximum puncture reaction force during skin breakthrough. This problem might also be solved by identifying puncture conditions that minimize skin deformation and needle bending.

\section{Reaction force during needle insertion}

Figures 9 and 10 shows that the several puncture events are designated by a peak in force after a steady rise, followed by a sharp decrease. These dataset explain that the tissues do not respond with a constant force when the needle punctures the tissue. Instead, the needle first pushes the tissue and then instantaneously punctures it. The decrease in the graph of Figures. 9 and 10 means that the needle punctured the several tissue (skin, fat, muscle, membrane, vessels etc.). In fact, the decrease of reaction force are observed before surgeon commanded to stop the needle. As discussed above, the stick-slip phenomenon produces a wavelike rise and fall in reaction force and it appears that the surgeon saw this waveform and mistook it as a sign of needle entry into the vein. We will develop the information technology to identify the needle ant tissue interaction situation using reaction force to solve these problem in future work.
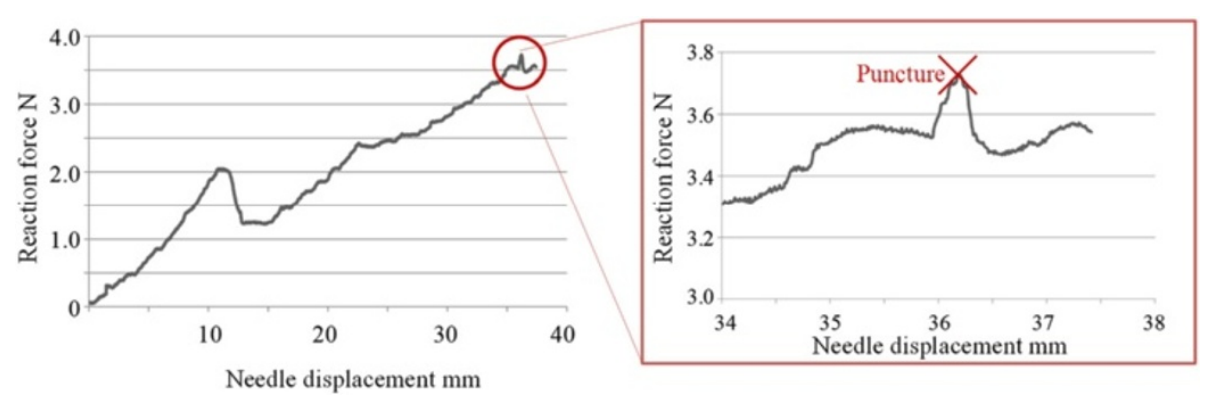

Figure 10 The relation between needle displacement and reaction force in the puncture through unopened skin. 


\section{Conclusion}

In an in vivo experiment conducted on a porcine, we evaluated the needle insertion manipulator developed in our previous research. The experimental results confirmed a high venous placement rate of $80 \%$ for puncture under the proposed conditions with the skin opened. Needle placement inside the vein was unsuccessful in one attempt, but this was because the surgeon stopped the needle in error in response to a sudden fall in reaction force before the vein was reached. This sudden fall could have been caused by stick-slip between the sides of the needle and the surrounding tissue. In the near future, we must therefore identify not only the conditions that facilitate needle placement in the vein, but also the puncture conditions that inhibit the stick-slip phenomenon, which is misinterpreted by the entry detection function. With the skin intact, the venous placement rate was $40 \%$, which is clearly poorer than that when the skin is opened. The primary causes for this low success rate were deviation from the puncture point secondary to the effects of the skin and bending of the needle, which lead to a mismatch between the planned and actual puncture routes so that the needle missed the target vein. We must attempt to solve this problem by identifying the puncture conditions that can best minimize needle bending due to the presence of skin. As proof of this problem, the accident rate in clinical practice is reportedly approximately $20 \%$. In summary, this study showed the effectiveness of using a puncture-assist manipulator for in vivo venous puncture in a porcine model with the skin opened.

\section{Competing interests}

The authors declare that they have no competing interests.

\section{Authors' contributions}

YK devised the basic concept of the overall system, technically constructed the system and drafted the manuscript. $\mathrm{RH}$ technically constructed the system, carried out the experiments and helped to draft the manuscript. HW and TK technically constructed the part of the system and carried out the experiments. JH devised the basic concept of the overall system. KT devised the research plan of in vivo experimental based on the engineering point of view. MU, SI, MT and TO devised the research plan of in vivo experimental based on the clinical point of view and gave the clinical discussion of the results. $\mathrm{MH}$ devised the basic concept and drew the research design based on the clinical point of view. MF devised the basic concept and drew the research design of the overall study. All authors read and approved the final manuscript.

\section{Sources of financial support}

This work was supported in part by the Global Centers of Excellence (COE) program "Global Robot Academia" from the Ministry of Education, Culture, Sports, Science and Technology of Japan; in part by a Grant-in-Aid for Challenging Exploratory Research (No. 20650078); in part by a Grant-in-Aid for Young Scientists (B) (Nos. 21700513 and 23700585); and in part by a Grant-in-Aid from Tateishi Science and Technology Foundation.

\section{Author details}

${ }^{1}$ Faculty of Science and Engineering \& Research Institute for Science and Engineering, Waseda University, 59-309, Ohkubo, Shijuku, Tolyo 169-8555, Japan. '2Department of Robotics Engineering, Daegu Gyeongbuk Institute of Science and Technology (DGIST), Daegu, Korea. ${ }^{3}$ Department of Advanced Medicine and Innovative Technology, Kyushu University Hospital, Fukuoka, Japan.
Received: 26 December 2013 Accepted: 19 October 2014

Published online: 16 November 2014

\section{References}

1. Fichtinger G, Deweese TL, Patriciu A, Tanace A, Mazilu D, Anderson JH, Masamune K, Taylor RH, Stoianovici D (2002) System for robotically assisted prostate biopsy and therapy with intraoperative $C T$ guidance. Academic Radiology 9(1):60-74

2. Masamune K, Fichtinger G, Patriciu A, Susil RC, Taylor RH, Kavoussi LR, Anderson JH, Sakuma I, Dohi T, Stoianovici D (2001) System for robotically assisted percutaneous procedures with computed tomography guidance. J Comp Aided Surgery 6(6):370-383

3. Loser MH, Navab N (1935) A new robotic system for visually controlled percutaneous interventions under CT fluoroscopy. MICCAI 2000:887-896

4. Yanof J, Haaga J, Klahr P, Bauer C, Nakamoto D, Chaturvedi A, Bruce R (2001) $\mathrm{CT}$-integrated robot for interventional procedures: preliminary experiment and computer-human interfaces. J Comp Aided Surgery 6:352-359

5. Masamune K, Ohara F, Matsumiya K, Liao H, Hashizume M, Dohi T (2006) MRI compatible robot for needle placement therapy with accurate registration. In: Proceedings of World Congress on Medical Physics and Biomedical Engineering, vol 14. pp 3056-3059

6. Hashizume M, Yasunaga T, Tanoue K, leiri S, Konishi K, Kishi K, Nakamoto H, Ikeda D, Sakuma I, Fujie M, Dohi T (2008) New real-time MR image-guided surgical robotic system for minimally

invasive precision surgery. Int J Comp Ass Radiology Surgery 2(6):317-325

7. Krieger A, Susil RC, Ménard C, Coleman JA, Fichtinger G, Atalar E, Whitcomb LL (2005) Design of a novel MRI compatible manipulator for image guided prostate interventions. IEEE Trans Biomed Eng 52(2):306-313

8. Terayama M, Furusho J, Monden M (2007) Curved multi-tube device for path-error correction in a needle-insertion system. In J Med Rob Comp Assisted Surgery 3:125-134

9. Fichtinger G, Fiene JP, Kennedy CW, Kronreif G, lordachita I, Song DY, Burdette EC, Kazanzides P (2008) Robotic assistance for ultrasound-guided prostate brachytherapy. Med Image Analysis 12(5):535-545

10. Boctor EM, Fichtinger G, Taylor RH, Choti MA (2003) Tracked 3D ultrasound in radio-frequency liver ablation. Medical Imaging 5035:174-182

11. Bassan H, Patel RV, Moallem M (2009) A novel manipulator for percutaneous needle insertion: design and experimentation. IEEE/ASME Trans Mechatronics 14:746-761

12. Hong J, Dohi T, Hashizume M, Konishi K, Hata N (2004) An ultrasound-driven needle insertion robot for percutaneous cholecystostomy. Physics Med Biology 49:441-455

13. Kobayashi Y, Onishi A, Watanabe H, Hoshi T, Kawamura K, Hashuzume M, Fujie MG (2010) Deformation simulation using a viscoelastic and nonlinear organ model for control of a needle insertion manipulator. In Journal of Comp Med Imaging Graph 34(1):9-18

14. Oura M, Kobayashi Y, Okamoto J, Fujie MG (2006) Development of MRI compatible versatile manipulator for minimally invasive surgery. In: Proceedings of 2006 IEEE Biomedical Robotics and Biomechatronics. pp 176-181

15. Kobayashi Y, Hong J, Hamano R, Okada K, Fujie MG, Hashizume M (2011) Development of a needle insertion manipulator for central venous catheterization. Int J Med Rob Comp Assisted Surgery 8(1):34-44

16. Kobayashi Y, Hamano R, Hong J, Watanabe H, Toyoda K, Hashizume M, Fujie MG (2013) Use of puncture force measurement to investigate the conditions of blood vessel needle insertion. Med Eng Physics 35(5):684-689

\section{doi:10.1186/s40648-014-0018-3}

Cite this article as: Kobayashi et al:: Preliminary in vivo evaluation of a needle insertion manipulator for central venous catheterization. ROBOMECH Journal 2014 1:18. 